\title{
Spin-orbit effects on the spin and pseudospin polarization in ac-driven silicene
}

\author{
Alexander López \\ Escuela Superior Politécnica del Litoral, ESPOL, Departamento de Física, \\ Campus Gustavo Galindo Km. 30.5 Vía Perimetral, \\ P. O. Box 09-01-5863, Guayaquil, Ecuadov* \\ Francisco Mireles \\ Departamento de Física Teórica, Centro de Nanociencias y Nanotecnología, \\ Universidad Nacional Autónoma de México, \\ Apdo. Postal 14, 22800 Ensenada B.C., México \\ John Schliemann \\ Institute of Theoretical Physics, University of Regensburg, \\ Universität Str. 31, 93053, Regensburg, Germany \\ Benjamin Santos \\ INRS - Énergie Matériaux Télécommunications, Varennes, QC J3X 1S2, Canada
}

(Dated: April 29, 2022) 


\begin{abstract}
We study the pseudospin and spin dynamical effects in single-layer silicene due to a perpendicular electric field periodically driven and its interplay with the intrinsic and extrinsic (Rashba) spin-orbit interaction. We find that the spin nonconserving processes of the real spin of the quasiparticles in silicene, - induced by the rather weak spin-orbit mechanisms -, manifest themselves as shifts of the resonances of its quasienergy spectrum in the low coupling regime to the driving field. We show that there is an interesting cooperative effect among the, in principle, competing Rashba and intrinsic spin-orbit contributions. This is explicitly illustrated by exact and approximated analytical solutions of the dynamical equations. In addition, we show that a finite Rashba spin-orbit interaction is indeed necessary in order to achieve a nonvanishing spin polarization. As additional feature, trivial and nontrivial topological phases might be distinguished from each other as fast or slow dynamical fluctuations of the spin polarization. We mention the possible experimental detection schemes of our theoretical results and their relevance in new practical implementation of periodically driven interactions in silicene physics and related two-dimensional systems.
\end{abstract}




\section{INTRODUCTION}

Silicene $e^{-\underline{\underline{5}}}$ is an interesting material belonging to the family of two-dimensional systems that support massive Dirac fermions within their low energy regime. As well as graphene $\mathrm{e}^{\underline{6}-\underline{\underline{8}}}$, its carbon counterpart, silicene has a honeycomb lattice structure with two interpenetrated triangular sublattices. Yet, in silicene, its lattice structure is naturally buckled. This in turn implies that fermions at each sublattice site will respond different to perpendicular applied electric fields. Within the static scenario, previous works have shown that applying a constant and perpendicular electric field, a topological phase transition can be realized $^{9}$. In addition, recent proposals that employ laser irradiation have shown to protect topological phases in Weyl semimetals ${ }^{10}$. Studies of silicene in the nonequilibrium regime have shown the realization of dynamical topological phases within the so-called off resonant approximation ${ }^{11}, 12$. Yet, these works neglected the Rashba spin-orbit interaction since it is typically much smaller than the other energy scales in the problem. We show in this work that nevertheless, even a rather weak Rashba interaction in silicene can induce a nontrivial spin polarization effect when a periodically varying electric field is perpendicularly applied to the sample and as long as the system is in the resonant regime.

The study is focused within the Kane and Mele formulation for Dirac fermions in the low energy approximation and investigate the spin-pseudospin effects due to a periodic time modulated perpendicular electric field $E_{\mathrm{z}}(t) \underline{13}$. We show that a necessary requirement for inducing a nontrivial dynamical evolution of physical observables, such as the spin polarization, does require the nonvanishing of the Rashba spin-orbit interaction. In this manner, our work differs from those previous approaches that have overlooked the role of this spin nonconserving interaction in silicene. In order to tackle the problem of silicene with periodically driven electric field, we make use of the standard Floquet's theorem approach $\underline{14}^{-16}$ and compare the exact numerical results with the rotating wave approximation (RWA) for experimentally achievable parameter regimes. We focus on the description of the physics close to resonance and near the critical value of the static field that induces an energy gap closing of its spectrum. As it is already well established in the literature, the behavior of the energy gap is important in order to determine the topological phase of the driven system

both in the static regime $\underline{17}^{-19}$ as well as in the driven scenario $\underline{20} \underline{26}$. We show that our results of the nontrivial spin dynamical effects are qualitatively dependent on the zero momentum 
bandgap.

The structure of the paper is as follows. In the next section we present the single particle Dirac-like model for silicene under the presence of a time-varying electric field and spin-orbit interaction and give numerical results for the quasienergy spectrum by full diagonalization of the Floquet Hamiltonian. These exact numerical results are compared to the approximate quasienergy spectra obtained via the RWA scheme for experimentally suitable parameter regimes. Then, in section III we evaluate the spin and pseudospin polarizations induced by the driving field using the analytical RWA solution which allows us in turn to gain a better physical insight on the relevance of the resonantly induced processes as well of the importance of the Rashba spin-orbit interaction. In section IV we present concluding remarks and argue on the experimental feasibility of the realization of our findings.

\section{MODEL}

We consider a monolayer silicene sample subject to a perpendicular static electric field $E_{\mathrm{s}}$. In addition, we consider a homogeneous time-dependent electric field component $E_{\mathrm{z}}(t)$ that drives the system out of equilibrium. Then, following references -11 the long wavelength (low energy) approximation leads to the $8 \times 8$ Dirac-like Hamiltonian $(\hbar=1)$,

$$
\begin{aligned}
\mathcal{H}_{\eta}(\vec{k}, t) & =v_{\mathrm{F}}\left(\eta k_{x} \sigma_{x}+k_{y} \sigma_{y}\right)+\eta s_{z} \sigma_{z} \lambda_{\mathrm{I}} \\
& +\eta h_{11} \sigma_{z}+h_{22}-\ell\left[E_{\mathrm{s}}+E_{\mathrm{z}}(t)\right] \sigma_{z},
\end{aligned}
$$

where $v_{\mathrm{F}}=\frac{\sqrt{3} a t_{b}}{2} \approx 10^{5} \mathrm{~m} / \mathrm{s}$ is the Fermi velocity for charge carriers in silicene with $a=3.86 \AA$ the lattice constant and $t_{\mathrm{b}}=1.6 \mathrm{eV}$ is the hopping parameter. Here $\ell=0.23 \AA$ measures half the separation among the two sublattice planes. In addition, $\eta= \pm 1$ describes the $K, K^{\prime}$ Dirac points, $\sigma_{i}$ and $s_{i}$ are Pauli matrices describing pseudo and real spin degrees of freedom respectively, whereas $\vec{k}=\left(k_{x}, k_{y}\right)$ is the in plane momentum measured from the Dirac point. The parameter $\lambda_{\mathrm{I}}=3.9 \mathrm{meV}$ represents the strength of the effective intrinsic spin-orbit contribution within a tight binding approximation. Furthermore, the two contributions given by the terms $h_{11}=a \lambda_{\mathrm{R} 2}\left(k_{y} s_{x}-k_{x} s_{y}\right)$ and $h_{22}=\lambda_{R 1}\left(\eta s_{y} \sigma_{x}-s_{x} \sigma_{y}\right) / 2$ describe the Rashba spin-orbit coupling associated to the nearest neighbor hopping and next-nearest neighbors, respectively. It is found that $\lambda_{R 2}=0.7 \mathrm{meV}$ and typically $h_{22}$ is much smaller than the other energy scales in the problem $\stackrel{9}{ }$. Thus, we keep $h_{11}$ and neglect $h_{22}$ in the 
following since our results will show that this Rashba spin-orbit contribution is a necessary ingredient for nontrivial manipulation of the spin polarizaton of Dirac fermions. Using the basis $\left\{\psi_{A \uparrow}, \psi_{B \uparrow}, \psi_{A \downarrow}, \psi_{B \downarrow}\right\}$, at the $\mathbf{K}$ Dirac point $(\eta=+1)$ we have the reduced Hamiltonian

$$
\mathcal{H}(\vec{k}, t)=\left(\begin{array}{cccc}
E_{-}(t) & v_{F} k_{-} & i v_{2} k_{-} & 0 \\
v_{F} k_{+} & -E_{-}(t) & 0 & -i v_{2} k_{-} \\
-i v_{2} k_{+} & 0 & -E_{+}(t) & v_{F} k_{-} \\
0 & i v_{2} k_{+} & v_{F} k_{+} & E_{+}(t)
\end{array}\right),
$$

where $k_{ \pm}=k_{x} \pm i k_{y}, E_{ \pm}(t)=\lambda_{I} \pm \ell\left[E_{s}+E_{z}(t)\right]$ and $v_{2}=a \lambda_{R 2}$. If we now define $\tan \phi=k_{y} / k_{x}$ and perform a unitary transformation with $\mathcal{H}_{1}(k, t)=\mathcal{R}_{\phi}^{\dagger} \mathcal{H}(\vec{k}, t) \mathcal{R}_{\phi}$ with $\mathcal{R}_{\phi}=\operatorname{Diag}\left(e^{-i \phi}, 1,1, e^{i \phi}\right)$ we get

$$
\mathcal{H}_{1}(k, t)=\left(\begin{array}{cccc}
E_{-}(t) & v_{F} k & i v_{2} k & 0 \\
v_{F} k & -E_{-}(t) & 0 & -i v_{2} k \\
-i v_{2} k & 0 & -E_{+}(t) & v_{F} k \\
0 & i v_{2} k & v_{F} k & E_{+}(t)
\end{array}\right) .
$$

To simplify the notation we will denote $\lambda_{R 2}=\lambda_{R}$ whenever we refer to the Rashba spin-orbit strength. Introducing the $4 \times 4$ matrix $\Lambda=s_{x} \otimes \mathbb{1}$, which explicitly reads

$$
\Lambda=\left(\begin{array}{ll}
0 & \mathbb{1} \\
\mathbb{1} & 0
\end{array}\right),
$$

with $\mathbb{1}$ being the $2 \times 2$ identity matrix, the Hamiltonian takes a block diagonal form

$$
\mathcal{H}_{2}(t)=\mathcal{T}_{\alpha}^{\dagger} \mathcal{H}(t) \mathcal{T}_{\alpha}=\left(\begin{array}{cc}
H_{2-}(t) & 0 \\
0 & H_{2+}(t)
\end{array}\right),
$$

where the unitary transformation has the explicit form $\mathcal{T}_{\alpha}=\exp (-i \alpha \Lambda / 2)$ and $\alpha$ is chosen to get rid of the off-diagonal terms. After some straightforward algebra one gets the condition for block diagonalization to fix the angle of transformation by the parameter relation $\tan \alpha=$ $v_{2} k / \lambda_{I}$. Then, we find that

$$
H_{2 \pm}(t)=\mp \Delta_{ \pm} \sigma_{z}+v_{F} k \sigma_{x}-\ell E_{z}(t) \sigma_{z},
$$

where we have introduced the momentum-dependent static bandgap $\Delta_{ \pm}=\sqrt{\lambda_{I}^{2}+\left(v_{2} k\right)^{2}} \pm$ $V_{z}$, with $V_{z}=\ell E_{\mathrm{s}}$. In absence of the Rashba term $\left(v_{2}=0\right)$, the static electric field has a 
critical value $E_{s}=E_{c} \equiv \lambda_{I} / \ell$ for which the static bandgap closes $\left(\Delta_{-}=0\right)$ at one of the Dirac cones. This is the so-called single-valley Dirac cone phase. Thus, in the vicinity of the band inversion, one would expect that the typically small Rashba term proportional to $v_{2}$ might have a significant role in the dynamics, at least at large momenta. This maximum value of the particle's momentum is, for consistency, determined by the validity of the linear dispersion approximation. For instance, in a typical scattering problem this maximum value corresponds to the Fermi momentum $k_{\mathrm{F}}$. We are interested in determining the interplay among the Rashba nonconserving spin processes and the modulation effects due to the driving field $\ell E_{z}(t)$, focusing in the near resonant parameter regime where the RWA holds. Before proceeding further we perform an additional unitary transformation $\mathcal{D}=\operatorname{Diag}\left\{D_{-}, D_{+}\right\}$, giving

$$
\mathcal{H}_{3}(t)=\mathcal{D}^{\dagger} \mathcal{H}_{2}(t) \mathcal{D}=\left(\begin{array}{cc}
H_{3-}(t) & 0 \\
0 & H_{3+}(t)
\end{array}\right)
$$

where $D_{ \pm}=\sigma_{z} \cos \frac{\gamma_{ \pm}}{2} \mp \sigma_{x} \sin \frac{\gamma_{ \pm}}{2}$ and we have defined $\tan \gamma_{ \pm}=v_{F} k / \Delta_{ \pm}$. We find then

$$
H_{3 \pm}(t)=\mp \omega_{ \pm} \sigma_{z}-\ell E_{z}(t)\left(\cos \gamma_{ \pm} \sigma_{z} \mp \sin \gamma_{ \pm} \sigma_{x}\right)
$$

where we have introduced the effective energy $\omega_{ \pm}=\sqrt{\left(v_{F} k\right)^{2}+\Delta_{ \pm}^{2}}$. In order to explore the single-valley Dirac cone scenario beyond the static regime we consider a periodic timedependent electric field $E_{z}(t+T)=E_{z}(t)$, where $T$ is the period. Then, both of the Hamiltonians $H_{3 \pm}(t)$ inherit its periodicity and we can resort to Floquet theorem $\underline{14} \underline{-16}$ which states that the general solution to the dynamics

$$
i \partial_{t} \mathcal{U}_{3}(t)=\mathcal{H}_{3}(t) \mathcal{U}_{3}(t)
$$

can be written as

$$
\mathcal{U}_{3}(t)=\mathcal{P}(t) e^{-i \mathcal{H}_{F} t}
$$

with $\mathcal{P}(t)$ periodic and $\mathcal{H}_{F}$ a constant matrix, respectively. The eigenvalues of $\mathcal{H}_{F}$ give the quasienergy spectrum of the periodically driven problem. In order to determine these quasienergies one standard approach consists of performing an expansion in the (infinite) eigenbasis of time periodic functions $F_{n}(t)=e^{i n \Omega t}$, where the frequency is defined as $\Omega=2 \pi / T$. The resulting infinite eigenvalue problem is solved by a truncation procedure to determine the Floquet exponents in such a way that adding more modes dot not 


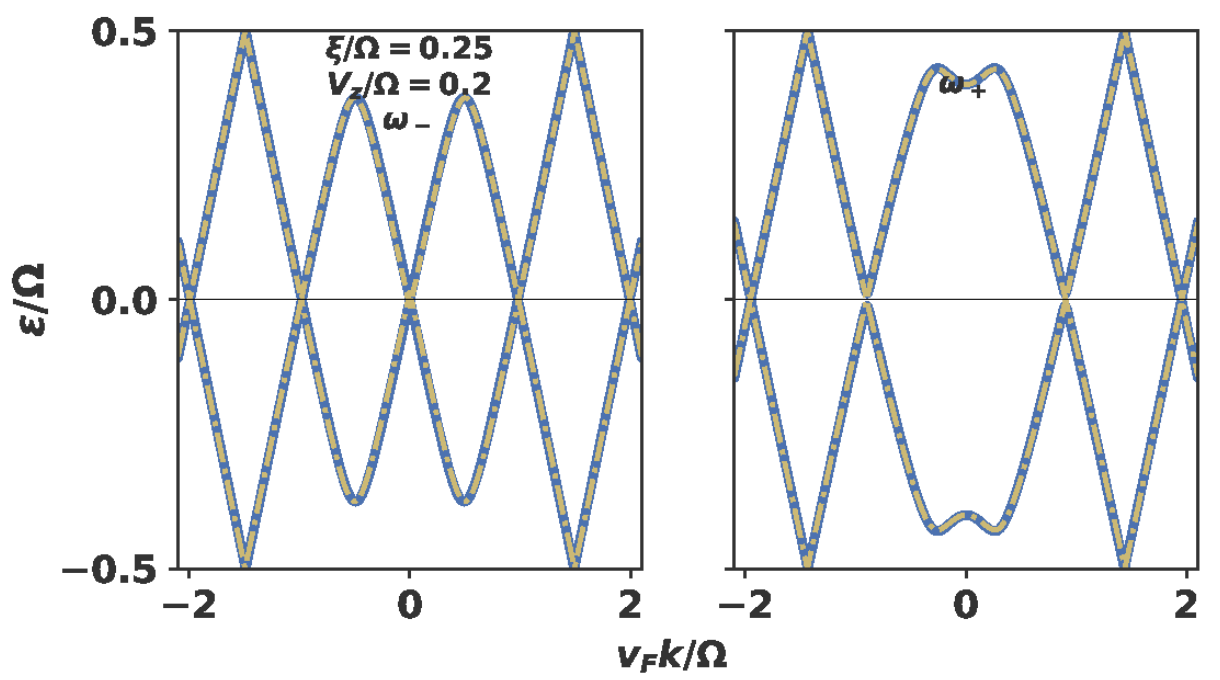

FIG. 1. (Color online) Exact (blue continuous line) and approximate (yellow dashed line) quasienergy spectrum for one of the effective spin components gapless (left panel) and the other gapped (right panel) at the Dirac cone $k_{x}=0$. This is the so-called single-valley Dirac cone configuration. The parameters have been chosen as $\xi=0.25 \Omega, \lambda_{I}=V_{z}=0.2 \Omega$ and $\lambda_{R}=0.05 \Omega$.

qualitatively modify the obtained quasienergies. In order to find the quasienergy spectrum one needs to evaluate the evolution operator at $t=T$ and diagonalize the resulting matrix $\mathcal{U}_{3}(T)$. In the following section we find an approximate semi-analytical solution to the energy spectrum and compare to the exact result obtained via numerical diagonalization of the Floquet Hamiltonian in Fourier representation. We also evaluate the dynamics of the spin polarization and show that a finite Rashba spin-orbit interaction contribution is necessary in order to obtain nontrivial dynamical effects on the spin polarization.

\section{ANALYTICAL RESULTS WITHIN THE RWA}

Let us now discuss an approximate solution leading to analytical expressions for the dynamical evolution of the physical quantities. This in turn allows us to highlight the main physical mechanisms underlying the spin and pseudospin control of the Dirac fermions.

We begin by rewriting the upper and lower components of the block diagonal Hamiltonian

$$
H_{3 \pm}(t)=\mp \omega_{ \pm} \sigma_{z}-\ell E_{z}(t)\left(\sigma_{z} \cos \gamma_{ \pm} \mp \sigma_{x} \sin \gamma_{ \pm}\right)
$$

where we recall that $\omega_{ \pm}=\sqrt{\left(v_{F} k\right)^{2}+\Delta_{ \pm}^{2}}$. Changing to the interaction representation the dynamics is dictated by $V_{I_{ \pm}}(t)=e^{\mp i \omega_{ \pm} t \sigma_{z}} V_{ \pm}(t) e^{ \pm i \omega_{ \pm} t \sigma_{z}}$, where $V_{ \pm}(t)$ is the time-dependent 
contribution shown in equation (11). Choosing the time dependence of the driving field as $\ell E(t)=\xi \sin \Omega t$ and using the algebra of the Pauli matrices we get,

$$
\begin{aligned}
V_{I_{ \pm}}(t)= & -\xi \sin \Omega t\left[\sigma_{z} \cos \gamma_{ \pm} \mp \sin \gamma_{ \pm}\right. \\
& \left.\left(\cos 2 \omega_{-} t \sigma_{x} \pm \sin 2 \omega_{-} t \sigma_{y}\right)\right]
\end{aligned}
$$

In order to implement the rotating wave approximation (RWA) we would like to remind that it is a standard approximation scheme that renders the Floquet Hamiltonian timeindependent by restricting to small values of the effective coupling strength to the driving field and for frequencies with values near those of the energies of the static Hamiltonian, i.e., near resonances. Hence, if we invoke the RWA, we get the approximate interaction contribution

$$
V_{I \pm}(t) \approx \frac{\xi \sin \gamma_{ \pm}}{2 i}\left(e^{\mp i \delta_{ \pm} t} \sigma_{+}-e^{ \pm i \delta_{ \pm} t} \sigma_{-}\right)
$$

where the frequency detuning that characterizes near resonance contributions is defined as $\delta_{ \pm}=2 \omega_{ \pm}-\Omega$. In addition, we have used the standard definitions $\sigma_{ \pm}=\left(\sigma_{x} \pm i \sigma_{y}\right) / 2$. To get (13) we have assumed $\Omega-2 \omega_{ \pm} \sim 0$ and thus have disregarded the quickly oscillating terms proportional to $\pm \Omega$ as well as those corresponding to the secular contributions $\Omega+2 \omega_{ \pm}$. Switching back to the Schrödinger representation we get the effective RWA Hamiltonian

$$
h_{ \pm}(t)=\mp \sigma_{z} \omega_{ \pm}-i g_{ \pm}\left(e^{ \pm i \Omega t} \sigma_{+}-e^{\mp i \Omega t} \sigma_{-}\right)
$$

where we have introduced the effective momentum-dependent coupling constant $g_{ \pm}$, that reads explicitly

$$
g_{ \pm}=\frac{\xi \sin \gamma_{ \pm}}{2}=\frac{\xi v_{F} k}{2 \omega_{ \pm}}
$$

The Hamiltonian (14) is taking to a time-independent form, $h_{F \pm}$, by means of periodic unitary transformations in each subblock matrix $P_{ \pm}(t)=e^{ \pm i\left(\mathbb{1}+\sigma_{z}\right) \Omega t / 2}$, which relies on the standard transformation rule $h_{F_{ \pm}}=P_{ \pm}^{\dagger}(t) h_{ \pm}(t) P_{ \pm}(t)-i P_{ \pm}^{\dagger}(t) \partial_{t} P_{ \pm}(t)$. Explicitly, we have

$$
h_{F_{ \pm}}= \pm \frac{\Omega}{2} \mathbb{1} \mp \frac{2 \omega_{ \pm}-\Omega}{2} \sigma_{z}+g_{ \pm} \sigma_{y}
$$

for which the approximate quasienergies are found to be given as

$$
\varepsilon_{\nu \pm}=\frac{1}{2}\left(\nu \sqrt{4 g_{ \pm}^{2}+\left(2 \omega_{ \pm}-\Omega\right)^{2}} \pm \Omega\right), \quad \bmod \Omega
$$


where $\nu= \pm 1$ is the energy sub-band index. Since the Hamiltonian preserves its in-plane rotational invariance, we set from now on $k_{y}=0$, without loss of generality and denote $k_{x}=k$. In FIG. 1 we show the quasienergy spectrum for the configuration when one spin component is gapped and the other gapless at $k=0$. The continuous line is the exact result obtained from a numerical diagonalization of both $H_{3 \pm}(t)$, whereas the dashed line corresponds to the RWA scheme for a set of parameters which has been set as $\lambda_{I}=V_{z}=0.2 \Omega$ and $\lambda_{R}=0.05 \Omega$. We find an excellent agreement between the RWA and exact results for an effective value of the coupling strength $\xi=0.25 \Omega$. Indeed, we have checked that the RWA properly describes the quasienergies even for values of the effective coupling strengh as large as $\xi=0.5 \Omega$ where they begin to differ from each other. If we choose the set of parameters $\lambda_{I}=0.2 \Omega$ and $V_{z}=0.1 \Omega$ with $\lambda_{R}=0.05 \Omega$ same coupling strength $\xi=0.25 \Omega$, we find that both pseudospin sectors are gapped at $k=0$, as it is shown in FIG. 2 .

Having checked the quasienergy spectrum, we turn our attention to the interplay of the Rashba spin-orbit coupling and driving field. Previous works have neglected the Rashba spin-orbit in silicene $e^{9,11,12}$ because it possesses a value much smaller than the other energy scales in the problem. Our purpose now is to show that it does have an important role in determining the time evolution of the spin polarization $S_{z}(t)$ and its interplay with the ac-driven component of the electric field renders nontrivial the time evolution of $S_{z}(t)$ and although small, we show that $\lambda_{R}$ is indeed a necessary ingredient for getting a finite $S_{z}(t)$ for a properly chosen initially prepared state. Our purpose is to show how the dynamics of the Dirac fermions can be modified via this parameter. Let us discuss these issues in detail. We first note that the block-diagonal structure of the Hamiltonian allows us to define an effective spin corresponding to these subblocks. Thus, we could expect that this block diagonal condition could imply that effective spin is an approximate conserved quantity. Yet, in the following we show this is not the case due to the competing effects of the Rashba spin-orbit interaction and the driving field. In order to quantify the robustness of spin polarization under the driving field and the spin nonconserving effects due to the Rashba spin-orbit coupling, we evaluate now the time evolution of the spin polarization $S_{z}(t)$, defined as

$$
S_{z}(t)=\left\langle\Psi(0)\left|\mathcal{U}^{\dagger}(t) \mathcal{S}_{z} \mathcal{U}(t)\right| \Psi(0)\right\rangle
$$

where $|\Psi(0)\rangle$ is the initially prepared electronic state and $\mathcal{S}_{z}=s_{z} \otimes \mathbb{1}$. The explicit form of the evolution operator $\mathcal{U}(t)$ would be given below. In order to highlight the role of the 


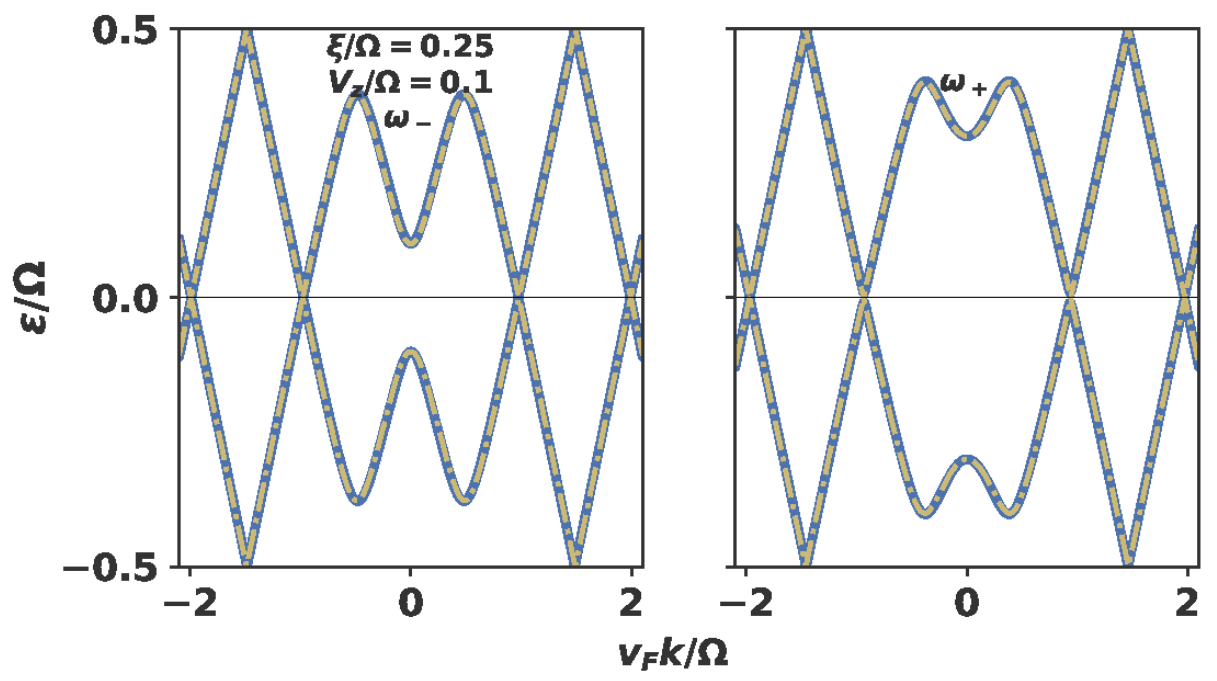

FIG. 2. (Color online) Exact (blue continuous line) and approximate (yellow dashed line) quasienergy spectrum for the two effective spin sectors being gapless at the Dirac cone $k=0$. The parameters have been chosen as $\xi=0.25 \Omega, \lambda_{I}=0.2 \Omega, V_{z}=0.1 \Omega$ and $\lambda_{R}=0.05 \Omega$.

driving field and Rashba term we will choose the initial state $|\Psi(0)\rangle$ as

$$
|\Psi(0)\rangle=\frac{1}{\sqrt{2}}\left(\begin{array}{c}
\left|\psi_{-}(0)\right\rangle \\
\left|\psi_{+}(0)\right\rangle
\end{array}\right)
$$

with vanishing initial spin polarization $S_{z}(0)=\left(\left\langle\psi_{-}(0) \mid \psi_{-}(0)\right\rangle-\left\langle\psi_{+}(0) \mid \psi_{+}(0)\right\rangle\right) / 2=0$. The simplest choice is to set $s_{z}\left|\psi_{ \pm}(0)\right\rangle= \pm\left|\psi_{ \pm}(0)\right\rangle$. Using the transformations that render the evolution operator block diagonal, it reads explicitly

$$
\mathcal{U}_{3}(t)=\left(\begin{array}{cc}
U_{3-}(t) & 0 \\
0 & U_{3+}(t)
\end{array}\right)
$$

with

$$
U_{3 \pm}(t)=\left(\begin{array}{cc}
a_{ \pm}(t) & b_{ \pm} \\
-b_{ \pm}^{*} & a_{ \pm}^{*}(t)
\end{array}\right)
$$

where the dimensionless functions $a_{ \pm}(t)$ and $b_{ \pm}(t)$ read for the RWA result as

$$
\begin{aligned}
& a_{ \pm}(t)=e^{ \pm i \Omega t / 2}\left[\cos \Gamma_{ \pm} t \pm \frac{i \delta_{ \pm} t}{2} \operatorname{sinc} \Gamma_{ \pm} t\right] \\
& b_{ \pm}(t)=g_{ \pm} t e^{ \pm i \Omega t / 2} \operatorname{sinc} \Gamma_{ \pm} t
\end{aligned}
$$

and satisfy the restriction

$$
\left|a_{ \pm}(t)\right|^{2}+\left|b_{ \pm}(t)\right|^{2}=1
$$



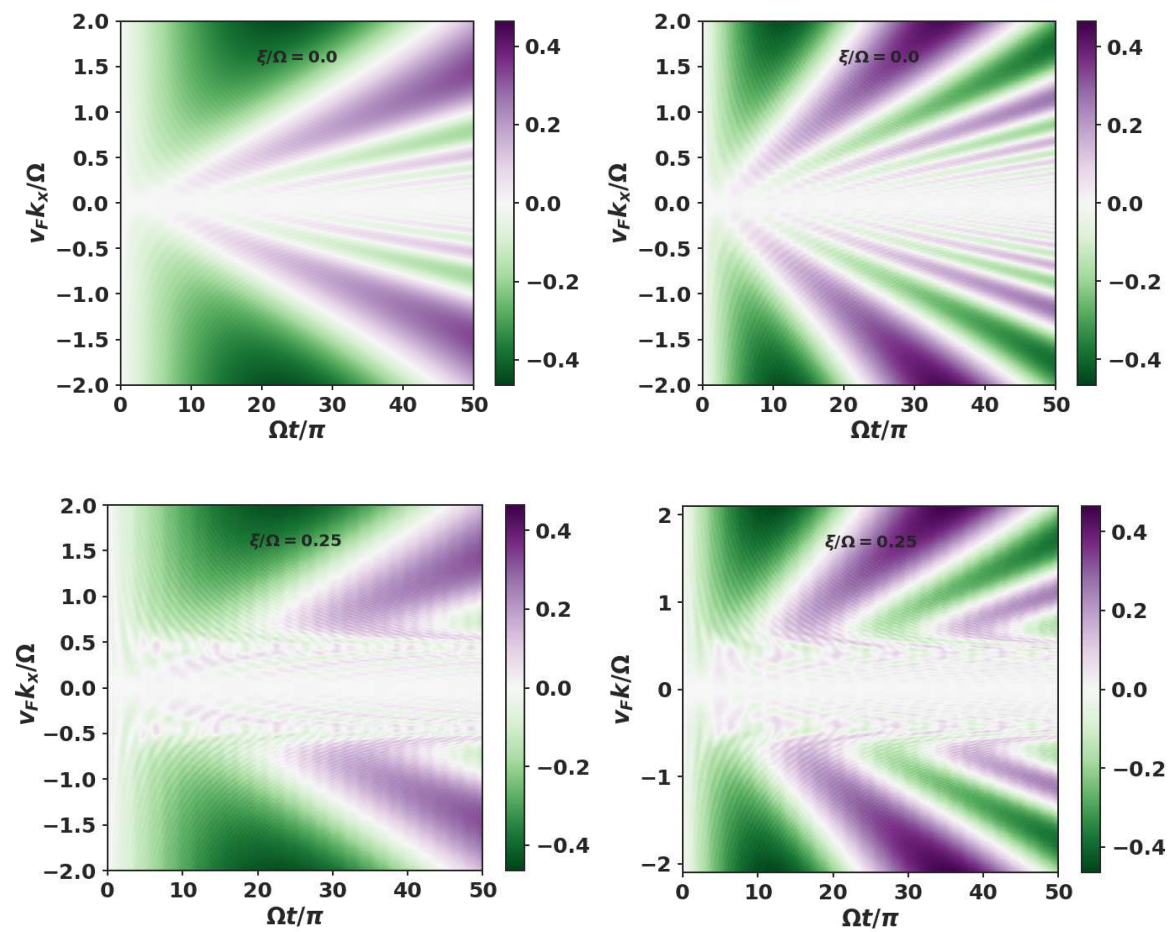

FIG. 3. (Color online) Effective spin polarization $S_{z}(t)$ as a function of time and momentum for $\lambda_{R}=0.05 \Omega$. The upper panels correspond to vanishing $\xi=0$ with left (right) panel having the other parameters as given in FIG. 1 (FIG. 2), respectively. The lower left (right) panel shows the $S_{z}(t)$ for finite $\xi=0.25 \Omega$ as in FIG. 1] (FIG. 2). We notice that near the Dirac point $(k=0)$ additional spin inversion flips signal the competing effects among the Rashba and driving field. For large momenta the Rashba contribution dominates and we observe no dynamical effects due to the driving field.

In addition, we have defined $\Gamma_{ \pm}=1 / 2 \sqrt{4 g_{ \pm}^{2}+\delta_{ \pm}^{2}}$, with $\delta_{ \pm}=2 \omega_{ \pm}-\Omega$ and we remind that $\tan \alpha=v_{2} k / \lambda_{I}$ measures the ratio of the two spin-orbit contributions that render the Hamiltonian block diagonal as was described previously. Thus we get

$$
\begin{aligned}
S_{z}(t) & =\frac{\sin \alpha}{2}\left\{\operatorname{Re}\left[W_{--}(t)-W_{++}(t)\right] \sin \alpha\right. \\
& \left.+\operatorname{Im}\left[W_{-+}(t)-W_{+-}(t)\right] \cos \alpha+\operatorname{Im}\left[W_{+-}(t)+W_{+-}(t)\right]\right\},
\end{aligned}
$$

where $W_{--}(t)=\left\langle\psi_{-}(0)|W(t)| \psi_{-}(0)\right\rangle$, and so on, are the matrix elements of the operator

$$
W(t)=D_{-} U_{3-}^{\dagger}(t) D_{-} D_{+} U_{3+}(t) D_{+} \cdot
$$


After some algebraic operations we get the result,

$$
\begin{aligned}
S_{z}(t)= & \sin \alpha \times \\
& {\left[\left(\operatorname{Im} a_{-} \operatorname{Re} b_{+}+\operatorname{Re} a_{+} \operatorname{Im} b_{-}\right) \cos \gamma_{-}\right.} \\
& \left(\operatorname{Im} b_{+} \operatorname{Re} a_{-}+\operatorname{Im} a_{+} \operatorname{Re} b_{-}\right) \cos \gamma_{+}+ \\
& \left(\operatorname{Re} b_{+} \operatorname{Im} b_{-}-\operatorname{Re} a_{+} \operatorname{Im} a_{-}\right) \sin \gamma_{-} \\
& \left.\left(\operatorname{Im} b_{+} \operatorname{Re} b_{-}-\operatorname{Im} a_{+} \operatorname{Re} a_{-}\right) \sin \gamma_{+}\right]
\end{aligned}
$$

Since the parameterization of the evolution operator given in equation (21) is valid in general, we must remark that the result given in equation (27) is exact and thus allows us to verify both, the numerical calculation and the RWA result. Indeed, the functional form of the dimensionless functions (22) for the exact result would differ from the relations corresponding to the RWA, but the polarization would still be calculated from expression (27), with the corresponding dimensionless quantities for the exact result. Since we have verified that RWA is valid for values of the coupling strength close to $\xi=0.5 \Omega$ we rely on the physics within the RWA which is encoded in the explicit form of the functions defined in equations (22). Moreover, the result given in equation (27) shows that the vanishing of the first term in the parenthesis of equation (25) means that all polarization effects are first order in the Rashba spin-orbit strength since $\sin \alpha \propto \lambda_{R}$ whereas the vanishing of the second term can be explained via the fact that it is proportional to the intrinsic spin-orbit interaction $\cos \alpha \propto \lambda_{I}$ which should not induce any spin-flipping processes.

Let us now discuss the results for the spin polarization $S_{z}(t)$ within the RWA as given in equation (27). In FIG. 3 we show a density plot of $S_{z}(t)$ as a function of momentum and time for different parameter configurations. The upper panels show the dynamical evolution of the polarization in absence of the driving field $(\xi=0)$ for the single-valley Dirac cone configuration FIG. 1 and gapless (FIG. 2) scenario. Yet, as seen in the two lower panels of FIG. 3, once $\xi=0.25 \Omega$ is finite a richer physical scenario is observed. In general, it is observed that spin-flipping processeses only require a finite value of the Rashba spin-orbit interaction and thus it should be considered as a valuable parameter in experimental setups. We would like to remark that previous works ${ }^{9}, 11,12$ have disregarded the role of the Rashba spin-orbit coupling in the physics of monolayer silicene based on its rather small value as compared to the other energy scales in the problem. However, our results for the dynamical evolution of the spin polarization show that for vanishing $\lambda_{R}$, there are not pseudospin-spin 
oscillations and $S_{z}(t)$ vanishes exactly for all values of the time and momentum variables for the initially chosen state. As discussed before, mathematically speaking, this can be seen by the $\sin \alpha$ prefactor in equation (27), but the physical reason for this result relies essentially in the nonconserving nature of the Rashba coupling. In presence of the driving field $(\xi=0.25 \Omega)$, the dynamics of $S_{z}(t)$ is determined by the effective spin sectors being in the configuration of FIG. 1 (lower left panel) or FIG. 2 (lower right panel) of the quasienergies shown with $V_{z}=\lambda_{I}\left(V_{z} \neq \lambda_{I}\right)$. In the first situation, the dynamics could be said to be fast, meaning that, within the same time window, more spin-flipping occurs for those quasienergies corresponding to the so-called single-valley Dirac cone configuration of FIG. $1 \mathbb{1}$. In this manner, the analysis of the dynamics of $S_{z}(t)$ could afford an experimental verification for the topological features of Dirac fermions in silicene and related materials. Moreover, for momenta in the vicinity of the Dirac point $k=0$, the role of the driving field is enhanced since it qualitatively modifies the time evolution of $S_{z}(t)$ which can in principle be understood as the resonant processes that lead to additional spin-flips and steems from the noncommuting nature of the Rashba spin-orbit interaction and the driving electric field and amounts to a direct manipulation of the pseudospin degree of freedom as we show below. Therefore, we find that even though the single-valley Dirac cone configuration $E_{z}=E_{c}$ might be expected as the only requirement for a nontrivial spin-pseudospin behavior of the Dirac fermions on a single layer of silicene, this is not the only parameter condition that renders nontrivial behavior on other quantities of interest, such as the spin polarization. Thus, our results show that indeed the, in principle, competing effects of both the Rashba term and the oscillating electric field are necessary in order to get a nontrivial evolution of the spin polarization. Of course, the Rashba term encodes the pseudospin-spin coupling and accounts for the angular momentum exchange among these two degrees of freedom whereas the resonant nature of the driven field is encoded in the $\Omega$ condition as well and the $\xi$ coupling strength that render the RWA valid.

In order to further explore the dynamical features of the model, we now focus on the pseudospin polarization dynamics, given as

$$
P_{z}(t)=\left\langle\Psi(0)\left|\mathcal{U}^{\dagger}(t) \mathcal{P}_{z} \mathcal{U}(t)\right| \Psi(0)\right\rangle
$$



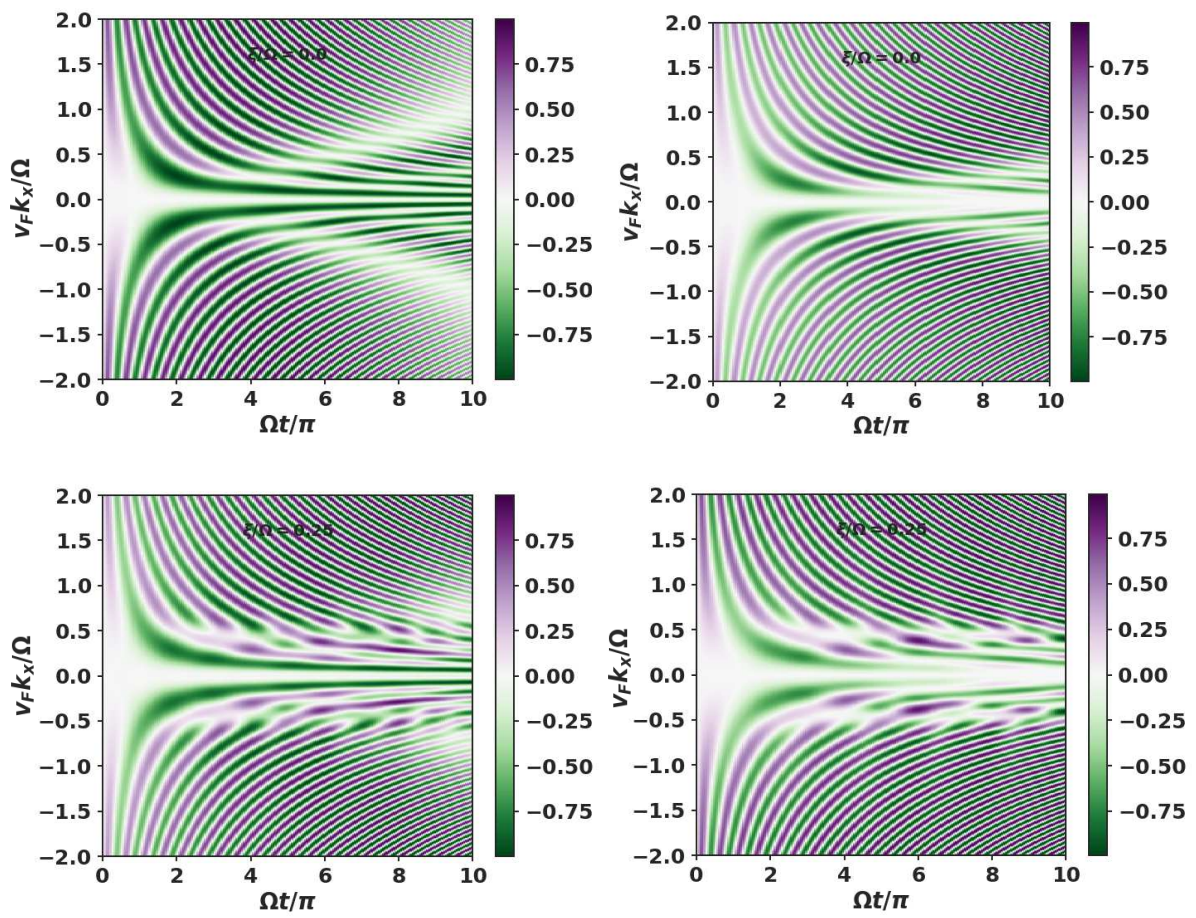

FIG. 4. (Color online) Effective pseudospin polarization $P_{z}(t)$ as a function of time and momentumfor the parameters chosen as in FIG. 3. Near the Dirac point $(k=0)$ the driving field effectively induces pseudospin inversion at finite driving.

with $\mathcal{P}_{z}=\mathbb{1} \otimes \sigma_{z}$. After some algebraic manipulations we get the result

$$
\begin{aligned}
P_{z}(t)= & \cos \alpha \times \\
& {\left[1 / 2\left(\cos ^{2} \gamma_{-}-\sin ^{2} \gamma_{-}\right)\left(\operatorname{Im} a_{-}^{2}-\operatorname{Im} b_{-}^{2}\right)+1 / 2\left(\operatorname{Re} a_{-}^{2}-\operatorname{Re} b_{-}^{2}\right)+\cos \gamma_{-} \sin \gamma_{-} \operatorname{Im} a_{-} \operatorname{Im} b_{-}\right.} \\
& \left.-1 / 2\left(\cos ^{2} \gamma_{+}-\sin ^{2} \gamma_{+}\right)\left(\operatorname{Im} a_{+}^{2}-\operatorname{Im} b_{+}^{2}\right)-1 / 2\left(\operatorname{Re} a_{+}^{2}-\operatorname{Re} b_{+}^{2}\right)-\cos \gamma_{+} \sin \gamma_{+} \operatorname{Im} a_{+} \operatorname{Im} b_{+}\right] \\
& -\sin \alpha \times \\
& {\left[\cos \gamma_{-}\left(\operatorname{Im} a_{-} \operatorname{Re} b_{-}-\operatorname{Im} b_{-} \operatorname{Re} a_{-}\right)+\sin \gamma_{-}\left(\operatorname{Im} a_{-} \operatorname{Re} a_{-}+\operatorname{Im} b_{-} \operatorname{Re} b_{-}\right)\right.} \\
& \left.-\cos \gamma_{+}\left(\operatorname{Im} a_{+} \operatorname{Re} b_{+}-\operatorname{Im} b_{+} \operatorname{Re} a_{+}\right)-\sin \gamma_{+}\left(\operatorname{Im} a_{+} \operatorname{Re} a_{+}-\operatorname{Im} b_{+} \operatorname{Re} b_{+}\right)\right], \quad(29)
\end{aligned}
$$

The results are shown in FIG. 4 for the same parameters as in fugure FIG.3. We note that contrary to what happens for $S_{z}(t)$, for vanishing value of the driving field strength $\xi=0$ (upper panels) show a qualitative behaviour which is not only dominated by the Rashba spinorbit interaction $\lambda_{R}=0.05 \Omega$. First we notice that the dynamics of $P_{z}(t)$ is much faster than that of $S_{z}(t)$ since more spin-flipping processes occur within a shorter time window. These might stem from the fact that even without Rashba spin-orbit interaction, the pseudospin 
is a nonconserved quantity since the static Hamiltonian is nondiagonal (even if $\lambda_{R}=0$ ) for finite momentum. In addition, in the lower panels (corresponding to finite driving) we notice that there is a more effective modulation of the pseudospin polarization. This is a natural consequence of the effective role of perpendicular electric fields acting differently at each sublattice in silicene. Moreover, from the figures in the lower panels of FIG. 4 we can infer that driving the system in the single-valley Dirac cone configuration of FIG. 1 (lower left panel) and the gapless scenario of FIG. 2(lower right panel) might be distinguished from each other by selecting those resonant procesesses happening in the vicinity of the resonances $v_{F} k / \Omega= \pm 0.5$ where pseudospin-flipping might be accounted for the avoiding crossings than can be observed around these values of momenta that qualitatively can be understood as an admixture of both pseudospin components with equal weights. We also notice in the lower left (right) panel that for small momenta, the long term dynamics $(\Omega t / \pi \geq 4)$ the trivial (nontrivial) topological scenario is dominated by vanishing (finite) value of the pseudospin close to zero momentum.

\section{CONCLUSIONS}

We have shown that the realization of effective spin-pseudospin exchange requires the interplay of both the spin nonconserving terms associated to the rather weak Rashba spin-orbit term as well as the time-dependent field strength. In fact, for the configuration where both of the effective spin sectors have an energy spectrum that is gapped at $k=0$, the cooperative effects of the Rashba term and the driving interaction are enhanced as the spin polarization shows additional flipping processes which require a shorter phase difference among the two spin components. Since this phase difference shifts the maxima and minima of $S_{z}(t)$, additional minima appear in its dynamical evolution. Indeed we could expect that the closer in value the quasienergy spectra of the two effective spin states are, the probability for spinflipping processes would in turn be reduced and the additional minima should disappear. We think that these findings could be interesting for a better understanding of the role of Rashba spin-orbit coupling in silicene and our results show that this previously disregarded spin interaction in silicene could indeed be accounted for in experimental setups where fine

control of the spin degree of freedom would be required. For an actual experimental realization, we could expect that standard spectroscopic techniques could allow the validation 
of our results, for instance via time-resolved spin-dependent photoemission measurements. We also have found that resonant processes play an important role in pseudospin dynamics and the evolution of these two quantities could afford a probe for the dynamical evolution and possible modulation of the topological properties of these new materials 27 for instance, by time-resolved ultrafast optical spectroscopy studies of topological insulators ${ }^{28}$ or by using time, spin and angle-resolved photoemission of the ultrafast dynamics of topological insulators $^{29}$ that can be extended to systems where large values of the Rashba spin-orbit interaction could be achieved in group IV films 30 .

\section{ACKNOWLEDGMENTS}

This work has been supported by Deutsche Forschungsgemeinschaft via GRK 1570 "Electronic Properties of Carbon Based Nanostructures". F. M. and A. L. acknowledge the support of PAPIIT-UNAM through the project IN111317.

* To whom correspondence should be addressed. Electronic address: alexander.lopez@physik.uni-regensburg.de

1 K. Takeda and K. Shiraishi, Phys. Rev. B 50, 14916 (1994).

2 G. G. Guzmán-Verri and L. C. Lew Yan Voon, Phys. Rev. B 76, 075131 (2007).

3 P. Vogt, P. De Padova, C. Quaresima, J. Avila, E. Frantzeskakis, M. C. Asensio, A. Resta, B. Ealet, and G. Le Lay, Phys. Rev. Lett. 108, 155501 (2012).

4 A. Fleurence, R. Friedlein, T. Ozaki, H. Kawai, Y. Wang, and Y. Yamada-Takamura, Phys. Rev. Lett. 108, 245501 (2012).

5 L. Chen, C.-C. Liu, B. Feng, X. He, P. Cheng, Z. Ding, S. Meng, Y. Yao, and K. Wu, Phys. Rev. Lett. 109, 056804 (2012).

6 K. S. Novoselov, A. K. Geim, S. V. Morozov, D. Jiang, Y. Zhang, S. V. Dubonos, I. V. Grigorieva, and A. A. Firsov, Science 306, 666 (2004).

7 A. K. Geim and K. S. Novoselov, Nature Materials 6, 183 (2007).

8 A. H. Castro Neto, F. Guinea, N. M. R. Peres, K. S. Novoselov, and A. K. Geim, Rev. Mod. Phys. 81, 109 (2009). 
9 M. Ezawa, Phys. Rev. Lett. 109, 055502 (2012).

10 J. González and R. A. Molina, Phys. Rev. B 96, 045437 (2017).

11 M. Ezawa, Phys. Rev. Lett. 110, 026603 (2013).

12 A. López, A. Scholz, B. Santos, and J. Schliemann, Phys. Rev. B 91, 125105 (2015)

13 C. L. Kane and E. J. Mele, Phys. Rev. Lett. 95, 226801 (2005).

14 J. H. Shirley, Phys. Rev. 138, B979 (1965).

15 M. Grifoni and P. Hnggi, Physics Reports 304, 229 (1998).

16 S.-I. Chu and D. A. Telnov, Physics Reports 390, 1 (2004).

17 C. L. Kane and E. J. Mele, Phys. Rev. Lett. 95, 146802 (2005).

18 M. Z. Hasan and C. L. Kane, Rev. Mod. Phys. 82, 3045 (2010).

19 B. A. Bernevig, T. L. Hughes, and S.-C. Zhang, Science 314, 1757 (2006).

20 T. Kitagawa, E. Berg, M. Rudner, and E. Demler, Phys. Rev. B 82, 235114 (2010).

21 N. H. Lindner, G. Refael, and V. Galitski, Nature Physics 7, 490 (2011).

22 T.-W. Chen, Z.-R. Xiao, D.-W. Chiou, and G.-Y. Guo, Phys. Rev. B 84, 165453 (2011).

23 J. Cayssol, B. Dóra, F. Simon, and R. Moessner, physica status solidi (RRL) Rapid Research Letters 7, 101 (2013).

24 T. Oka and H. Aoki, Phys. Rev. B 79, 081406 (2009).

25 Y. Zhou and M. W. Wu, Phys. Rev. B 83, 245436 (2011).

26 H. L. Calvo, H. M. Pastawski, S. Roche, and L. E. F. F. Torres, Appl. Phys. Lett. 98, 232103 (2011).

27 C. Cacho, A. Crepaldi, M. Battiato, J. Braun, F. Cilento, M. Zacchigna, M. Richter, O. Heckmann, E. Springate, Y. Liu, S. Dhesi, H. Berger, P. Bugnon, K. Held, M. Grioni, H. Ebert, K. Hricovini, J. Minár, and F. Parmigiani, Phys. Rev. Lett. 114, 097401 (2015).

28 M. Wang, S. Qiao, Z. Jiang, S. Luo, and J. Qi, Phys. Rev. Lett. 116, 036601 (2016).

29 J. Sánchez-Barriga, M. Battiato, M. Krivenkov, E. Golias, A. Varykhalov, A. Romualdi, L. V. Yashina, J. Minár, O. Kornilov, H. Ebert, K. Held, and J. Braun, Phys. Rev. B 95, 125405 (2017).

30 S.-j. Zhang, W.-x. Ji, C.-w. Zhang, P. Li, and P.-j. Wang, Scientific Reports 7, 45923 (2017). 